\title{
Association between expression of human papillomavirus 16/18 E6 oncoprotein and survival in patients with stage I non-small cell lung cancer
}

\author{
NAN-YUNG HSU ${ }^{1,7}$, YA-WEN CHENG ${ }^{2}$, I-PING CHAN ${ }^{3}$, HENG-CHIEN HO ${ }^{4}$, CHIH-YI CHEN $^{1}$, \\ CHUNG-PING HSU ${ }^{5}$, MENG-HUNG LIN ${ }^{6}$ and MING-CHIH $\mathrm{CHOU}^{2}$ \\ ${ }^{1}$ Division of Chest Surgery, China Medical University Hospital; ${ }^{2}$ Institute of Medicine, Chung Shan \\ Medical University; ${ }^{3}$ Department of Pathology, China Medical University Hospital; ${ }^{4}$ Department of Biochemistry, \\ College of Medicine, China Medical University; ${ }^{5}$ Division of Thoracic Surgery, Taichung Veterans General \\ Hospital; ${ }^{6}$ Graduate Institute of Environmental Health, China Medical University, Taichung, Taiwan, R.O.C.
}

Received August 11,2008; Accepted October 6, 2008

DOI: 10.3892/or_00000192

\begin{abstract}
The clinical significance of HPV-16/18 E6 oncoprotein expression in non-small cell lung cancer (NSCLC) is not fully known. A study was undertaken to investigate the association between expression of human papillomavirus 16/18 E6 oncoprotein and survival in patients with stage I NSCLC. We analyzed a series of 217 patients with stage I NSCLC for the presence of HPV-16/18 E6 oncoprotein by immunohistochemistry. HPV-16 E6 onco-protein was expressed in $49(22.6 \%)$ patients and HPV-18 E6 oncoprotein was expressed in $31(14.3 \%)$ patients. Statistical analysis revealed that the prevalence of expression of HPV-16 and HPV-18 E6 oncoproteins was significantly high in female patients, nonsmokers and patients with adenocarcinoma. The adjusted odds ratio for expression of HPV-16 E6 oncoprotein in female patients was 2.275 [95\% confidence interval (CI), 0.999-5.179] and that in patients with adenocarcinoma was 2.320 (95\% CI, 1.029-5.232). These ratios were significantly higher than those in male patients and patients with squamous cell carcinoma. Interestingly, we found that the 17 patients who expressed HPV-16 and HPV-18 E6 oncoprotein had a higher 5-year cumulate survival rate $(72.2 \%)$ than the 154 patients who did not express both oncoproteins $(48.3 \%)$; the difference was significant $(\mathrm{p}=0.055)$. Expression of HPV-16/18 E6 oncoprotein in stage I NSCLC may play an important role in female adenocarcinoma patients and survival benefits in patients who expressed HPV-16 and HPV-18 E6 oncoprotein.
\end{abstract}

Correspondence to: Dr Ya-Wen Cheng, Institute of Medicine, Chung Shan Medical University, No. 110, Sec. 1, Chien-Kuo N. Rd., Taichung 40203, Taiwan, R.O.C.

E-mail: h440506@ms15.hinet.net

Present address: ${ }^{7}$ Div. of Chest Surgery, Buddhist Tzu Chi General Hospital, Taichung Branch, Taiwan

Key words: human papillomavirus, non-small cell lung cancer

\section{Introduction}

Lung cancer is the most common cause of cancer-related deaths in the world, causing more than one million deaths worldwide each year (1). Pathological stage I non-small cell lung cancer (NSCLC) represents the fastest growing segment of patients with lung cancer because of the frequent use of high-resolution CT scan for screening (2). Although the prognosis is best for patients with pathological stage I NSCLC, the 5-year survival rate among these patients is only $57-67 \%$ (3). Histopathologic findings alone are insufficient to predict disease progression and clinical outcome. Factors that predict poor outcome in patients with stage I disease include the presence of the K-ras oncogene (4), a high ratio of type IV collagenases to E-cadherin (5), a low level of bcl-2 protein (6), and the loss of blood-group antigen A (7). However, none of these factors can be clinically detected, and no medical strategy has been developed to target these biomarkers. Thus, a multifactorial process depending on demographic, clinical, and biological characteristics should be sought to further improve survival in patients with pathological stage I NSCLC.

In our preliminary study, we found that human papillomavirus (HPV) infection is associated with lung cancer development in nonsmoking female patients (8). In contrast to studies that have demonstrated absence or low frequency of HPV infection in lung tumors (9-11), higher frequencies of HPV infection (25.6-78.3\%) in the same malignancy have been observed in Japan, Northern Iran, Finland and Norway (12-15). The association between HPV infection and lung cancer therefore has been suggested to be geographically- and race-dependent (8).

E6 proteins encoded by HPV bind to the host cellular tumor suppressor protein, $\mathrm{p} 53$, and induce its degradation through the ubiquitin pathway $(16,17)$. Thus, inactivation of p53 by HPV E6 is considered to play a crucial role in human carcinogenesis. Kinshoshita et al reported that HPV-18 E6 mRNA was detected in squamous cell carcinoma (SCC) $(1 / 10$, $10 \%)$ and adenocarcinoma of the lung $(2 / 22,9 \%)$, while no 
HPV-16 E6 mRNA was found in any of the 32 cases of lung cancer examined (18). Ciotti et al reported that 5 out of 6 fresh NSCLC specimens contained the E6 transript by sequencing the entire E6 oncogene of HPV-16 (19). Although Brouchet et al reported that none of the 122 cases of lung cancer containing a single positive tumor cell was found using anti-VP1 capsid protein (clone K1H8) (20), few studies of HPV-16/18 E6 oncoprotein expression in lung cancer have been conducted using immunohistochemistry. By using immunohistochemistry, we examined the expression of HPV16/18 E6 oncoprotein in 217 pathological stage I NSCLC patients and evaluated the difference between the clinicopathological variables and the expression of E6 oncoprotein. We also examined the clinical outcomes of stage I NSCLC patients who expressed the HPV-16/18 E6 oncoprotein.

\section{Materials and methods}

Study subjects. From January 2000 to December 2006, a total of 217 consecutive patients underwent surgical treatment for NSCLC at the China Medical University Hospital, Taichung, and at the Taichung Veterans General Hospital. Patients who had preoperative chemotherapy or radiotherapy were excluded from this study. Written informed consent to use paraffinembedded tissues and information regarding sociodemographic characteristics, as approved by the Institutional Review Board at the above two hospitals, was obtained from each patient before surgery. All of the available paraffin blocks were reviewed by a thoracic pathologist.

The study population consisted of 148 men and 69 women (mean age, 65.2 years; range, 37-83 years). Surgical treatment for NSCLC comprised pneumonectomy $(n=3 ; 1.4 \%)$, bilobectomy $(n=12 ; 5.5 \%)$, lobectomy $(n=180 ; 83.0 \%)$ and wedge resection $(n=22 ; 10.1 \%)$. All procedures included sampling of hilar and mediastinal lymph nodes, and pathology of all specimens confirmed stage I (T1-2N0M0) in all patients (3). Histological classification and grade were assessed by light microscopy according to WHO criteria (21). Clinical data including sex, age ( $\leq 65$ years vs. $>65$ years), smoking habit, histopathology (SCC vs. adenocarcinoma), tumor stage by TNM (T1 versus T2) and tumor differentiation were collected from patient charts and are shown in Table I.

Post-operative follow-up was scheduled at 1,2, and every 3 months during the first 2 years after surgery, and every 6 months thereafter, or more frequently if needed. The median duration of follow-up after a curative resection was 4.7 years.

Immunohistochemistry. All sections of formalin fixed and paraffin-embedded specimens were deparaffinized in xylene, rehydrated through serial dilutions of alcohol, and washed with PBS (pH 7.2). For HPV-16/18 E6 detection, sections were heated in a microwave oven twice for $5 \mathrm{~min}$ in citrate buffer ( $\mathrm{pH}$ 6.0), and then incubated with polyclonal antibodies raised against HPV-16/18 E6 (Santa Cruz, CA, USA and Chemicon International, Inc., CA, USA) for $90 \mathrm{~min}$ at $25^{\circ} \mathrm{C}$. The conventional streptavidin peroxidase method (Dako, LSAB kit K675, Copenhagen, Denmark) was performed to develop signals and the cells were counterstained with hematoxylin. The intensities of signals were evaluated independently by three observers. Negative
Table I. Clinical data of study subjects.

\begin{tabular}{lr}
\hline Variable & Frequency \\
\hline Gender & \\
Male & $148(68.2 \%)$ \\
Female & $69(31.8 \%)$ \\
Age, years & \\
$\leq 65$ & $95(43.8 \%)$ \\
$>65$ & $122(56.2 \%)$ \\
Smoking habit & \\
Yes & $87(40.1 \%)$ \\
No & $130(59.9 \%)$ \\
Tumor stage by TNM & \\
T1 & $33(15.2 \%)$ \\
T2 & $184(84.8 \%)$ \\
Histopathology & \\
SCC & \\
AD & $88(40.6 \%)$ \\
Tumor differentiation & $129(59.4 \%)$ \\
WD & \\
MD & \\
PD & $8(7.5 \%)$ \\
\hline
\end{tabular}

SCC, squamous cell carcinoma; AD, adenocarcinoma; WD, well differentiation; MD, moderate differentiation; PD, poor differentiation.

immunostaining was defined as $0-10 \%$ positive nuclei; positive immunostaining was defined as $>10 \%$ positive nuclei. The cervical cancer tumor tissues with HPV-16/18 were used as positive control for HPV16/18 E6. The antibody dilution buffer used to replace antibodies served as the negative control.

Statistical analysis. The significance of gender, age, histopathology, tumor stage by TNM, and tumor differentiation for the expression of E6 oncoprotein of HPV-16/18 was analyzed by the Chi-square test. Multiple logistic regression analysis was used to assess which variables were important for expression of E6 oncoprotein of HPV-16/18 and which factors might act as risk factors. Survival plots were generated using the Kaplan-Meier method and differences between patient groups were determined by the log-rank test. All reported $\mathrm{p}$-values are two-sided; $\mathrm{p}<0.05$ was considered significant.

\section{Results}

Fig. 1 shows representative samples of HPV-16/18 E6 oncoprotein expression in NSCLC. HPV16 E6 oncoprotein was expressed in 49 (22.6\%) patients and HPV18 E6 oncoprotein was expressed in $31(14.3 \%)$ patients (Table II). Statistical 
Table II. Association of expression of HPV-16/18 E6 oncoprotein with clinicopathological variables.

\begin{tabular}{|c|c|c|c|c|c|c|}
\hline \multirow[b]{2}{*}{ Variable } & \multicolumn{2}{|c|}{ HPV-16 E6 } & \multirow[b]{2}{*}{ p-value } & \multicolumn{2}{|c|}{ HPV-18 E6 } & \multirow[b]{2}{*}{ p-value } \\
\hline & $\begin{array}{c}\text { Positive } \\
49(22.6 \%)\end{array}$ & $\begin{array}{c}\text { Negative } \\
168(77.4 \%)\end{array}$ & & $\begin{array}{c}\text { Positive } \\
31(14.3 \%)\end{array}$ & $\begin{array}{c}\text { Negative } \\
186(85.7 \%)\end{array}$ & \\
\hline Gender & & & 0.002 & & & 0.006 \\
\hline Male & 24 & 124 & & 14 & 134 & \\
\hline Female & 25 & 44 & & 17 & 52 & \\
\hline Age, years & & & 0.513 & & & 0.117 \\
\hline$\leq 65$ & 19 & 76 & & 18 & 77 & \\
\hline$>65$ & 30 & 92 & & 13 & 109 & \\
\hline Smoking habit & & & 0.013 & & & 0.047 \\
\hline Yes & 12 & 75 & & 7 & 80 & \\
\hline No & 37 & 93 & & 24 & 106 & \\
\hline Tumor stage by TNM & & & 0.367 & & & 0.588 \\
\hline $\mathrm{T} 1$ & 5 & 28 & & 6 & 27 & \\
\hline $\mathrm{T} 2$ & 44 & 140 & & 25 & 159 & \\
\hline Histopathology & & & 0.005 & & & 0.010 \\
\hline SCC & 11 & 77 & & 6 & 82 & \\
\hline $\mathrm{AD}$ & 38 & 91 & & 25 & 104 & \\
\hline Tumor differentiaton & & & 0.924 & & & 0.375 \\
\hline WD & 2 & 6 & & 1 & 7 & \\
\hline MD & 15 & 63 & & 13 & 65 & \\
\hline $\mathrm{PD}$ & 4 & 17 & & 1 & 21 & \\
\hline
\end{tabular}

SCC, squamous cell carcinoma; AD, adenocarcinoma; WD, well differentiation; MD, moderate differentiation; PD, poor differentiation.

Table III. Crude odds ratio (OR) and adjusted OR of HPV-16 E6 expression in clinicopathological variables.

\begin{tabular}{lcccc}
\hline Variable & Crude OR & $95 \% \mathrm{CI}$ & Adjusted OR & $95 \% \mathrm{CI}$ \\
\hline Gender & & & & \\
$\quad$ Male & Ref & - & Ref & - \\
Female & 2.959 & $(1.534-5.709)^{\mathrm{b}}$ & 2.275 & $(0.999-5.179)^{\mathrm{b}}$ \\
Age, years & & & & - \\
$\quad \leq 65$ & Ref & - & Ref & $(0.888-3.609)$ \\
$>65$ & 1.290 & $(0.674-2.471)$ & 1.800 & - \\
Smoking habit & & & & $(0.519-3.183)$ \\
Yes & Ref & - & Ref \\
No & 2.460 & $(1.199-5.046)^{\mathrm{b}}$ & 1.279 & - \\
Tumor stage by TNM & & & & $(0.631-5.185)$ \\
T1 & Ref & - & Ref \\
T2 & 1.748 & $(0.636-4.798)$ & 1.808 & - \\
Histopathology & & & & $(1.029-5.232)^{\mathrm{b}}$ \\
SCC & Ref & - & Ref & \\
AD & 2.891 & $(1.385-6.037)^{\mathrm{b}}$ & 2.320 & \\
\hline
\end{tabular}


A

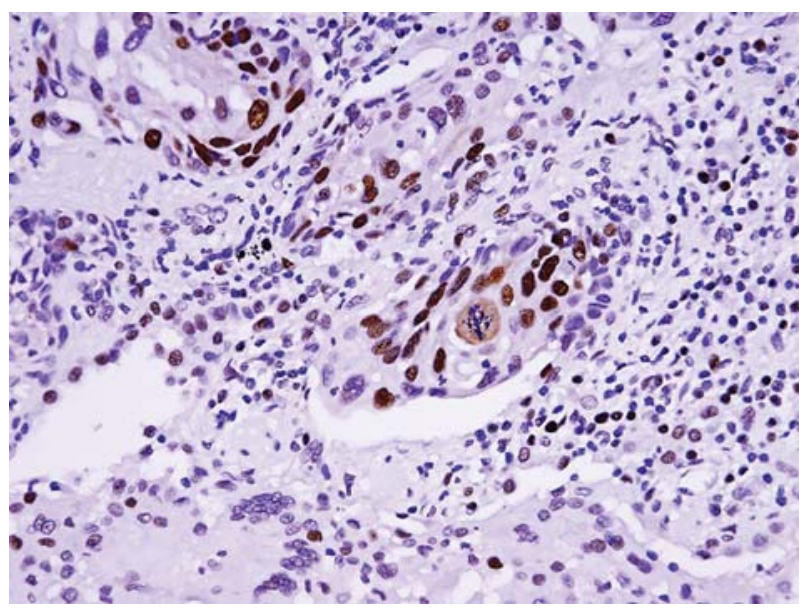

B

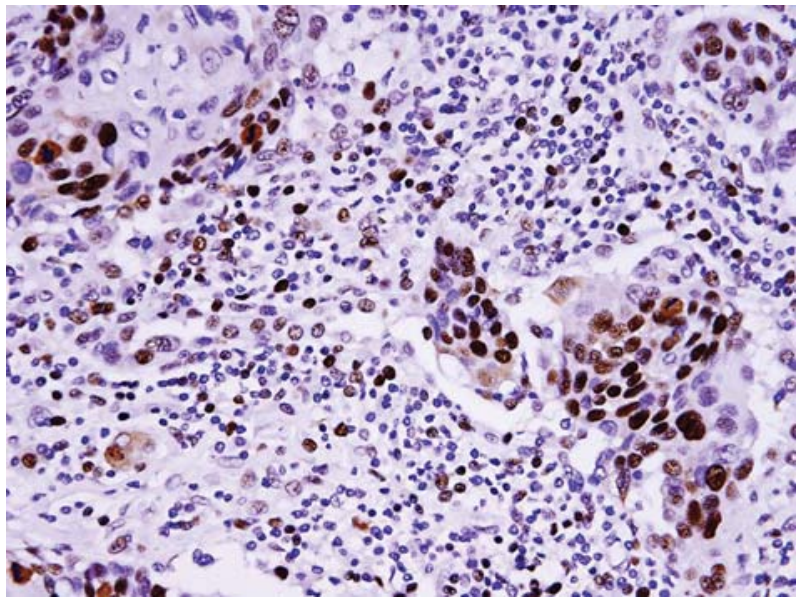

Figure 1. Representative findings on immunohistochemical staining of formalin-fixed paraffin-embedded cell pellets from specimens of patients with pathological stage I NSCLC. Cells from a sample of adenocarcinoma with expression of E6 oncoprotein of HPV-16 (A) (x400) and HPV-18 (B) (x400).

Table IV. Crude odds ratio (OR) and adjusted OR of HPV-18 E6 expression in clinicopathological variables.

\begin{tabular}{lcccc}
\hline Variable & Crude OR & $95 \%$ CI & Adjusted OR & $95 \%$ CI \\
\hline Gender & & & & \\
$\quad$ Male & Ref & - & Ref & - \\
Female & 2.921 & $(1.360-6.274)^{\mathrm{b}}$ & 1.887 & $(0.742-4.797)$ \\
Age, years & & & & - \\
$\quad$ 65 & Ref & - & Ref & $(0.324-1.578)$ \\
$>65$ & 0.549 & $(0.258-1.171)$ & 0.715 & - \\
Smoking habit & & & & $(0.444-3.965)$ \\
Yes & Ref & - & Ref \\
No & 2.695 & $(1.110-6.544)^{\mathrm{b}}$ & 1.327 & - \\
Tumor stage by TNM & & & & $(0.279-2.137)$ \\
T1 & Ref & - & Ref & \\
T2 & 0.736 & $(0.277-1.955)$ & 0.773 & - \\
Histopathology & & & & \\
SCC & Ref & - & Ref & $(0.845-6.415)$ \\
AD & 3.417 & $(1.341-8.692)^{\mathrm{b}}$ & 2.328 & \\
\hline
\end{tabular}

SCC, squamous cell carcinoma; AD, adenocarcinoma. ${ }^{\mathrm{a} A d j u s t e d ~ f o r ~ o t h e r ~ v a r i a b l e s ~ i n ~ t h i s ~ t a b l e . ~ R e f, ~ r e f e r e n c e ~ g r o u p ; ~}{ }^{\mathrm{b}} \mathrm{p}<0.05$.

analysis revealed that the expression of E6 oncoprotein of HPV-16 and -18 was significantly higher in female patients than in male patients ( $p=0.002$ for HPV -16 and $p=0.006$ for HPV-18). Statistical analysis also revealed that the expression of E6 oncoprotein of HPV-16 and -18 was significantly higher in nonsmokers $(\mathrm{p}=0.013$ for HPV-16 and $\mathrm{p}=0.047$ for HPV-18), and in patients with adenocarcinoma ( $\mathrm{p}=0.005$ for HPV-16 and $\mathrm{p}=0.01$ for HPV-18). Female patients had a significantly higher odd ratio (OR) of HPV-16 E6 oncoprotein expression (95\% confidence interval (CI), 1.534-5.709; $\mathrm{p}<0.05)$ than male patients; nonsmokers had a significantly higher OR of HPV-16 E6 oncoprotein expression (95\% CI, 1.199-5.046; $\mathrm{p}<0.05$ ) than smokers; and patients with adenocarcinoma had a significantly higher OR of HPV-16 E6 oncoprotein expression (95\% CI, 1.385-6.037; p<0.05) than patients with SCC. However, after adjusting for gender, age, smoking habit, tumor stage by TNM and histopathology variables, female patients had a significantly higher OR of HPV-16 E6 oncoprotein expression (95\% CI, 0.999-5.179; $\mathrm{p}<0.05)$ than male patients and patients with adenocarcinoma 
Table V. Univariate analysis of survival of clinicopathological variables and expression of HPV-16 E6 and -18 E6.

\begin{tabular}{|c|c|c|}
\hline Variable & $\begin{array}{l}\text { 5-year cumulate } \\
\text { survival rate }(\%)\end{array}$ & p-value \\
\hline Gender & & 0.013 \\
\hline Male & 45.6 & \\
\hline Female & 63.3 & \\
\hline Age, years & & 0.040 \\
\hline$\leq 65$ & 60.9 & \\
\hline$>65$ & 43.3 & \\
\hline Smoking habit & & 0.460 \\
\hline Yes & 47.9 & \\
\hline No & 53.7 & \\
\hline Tumor stage by TNM & & 0.308 \\
\hline $\mathrm{T} 1$ & 64.1 & \\
\hline $\mathrm{T} 2$ & 49.3 & \\
\hline Histopathology & & 0.170 \\
\hline SCC & 45.6 & \\
\hline $\mathrm{AD}$ & 55.3 & \\
\hline HPV-16 E6 & & 0.293 \\
\hline Expression & 57.6 & \\
\hline Non-expression & 49.0 & \\
\hline HPV-18 E6 & & 0.085 \\
\hline Expression & 65.2 & \\
\hline Non-expression & 48.5 & \\
\hline HPV-16 E6 and -18 E6 & & 0.055 \\
\hline Expression & 72.2 & \\
\hline Non-expression & 48.3 & \\
\hline
\end{tabular}

SCC, squamous cell carcinoma; AD, adenocarcinoma.

had a higher OR $(95 \%$ CI, 1.029-5.232; p<0.05) than patients with SCC (Table III).

Table IV shows that female patients had significantly higher odds of HPV-18 E6 oncoprotein expression (OR, 2.921 ; 95\% CI, 1.360-6.274; p<0.05) than male patients; nonsmokers had significantly higher odds of HPV-18 E6 oncoprotein expression, (OR, 2.695; 95\% CI, 1.110-6.544; $\mathrm{p}<0.05)$ than smokers; and patients with adenocarcinoma had significantly higher odds of HPV-18 E6 oncoprotein expression (OR, 3.417; 95\% CI, 1.341-8.692; p<0.05) than patients with SCC. However, after adjusting for sex, age, smoking habit, tumor stage and pathology, none of the variables were significant for HPV-18 E6 oncoprotein expression.

Univariate analysis of survival showed that female patients and age $\leq 65$ years were prognostic factors (Table V). Although patients with HPV-16 or -18 E6 oncoprotein expression had better survival outcomes than those lacking expression of either oncoprotein, there was no significant



Figure 2. Cox regression survival curve for patients with HPV-16 E6 ${ }^{+}$ /HPV-18 E6 ${ }^{+}(\mathrm{n}=17)$ and with HPV-16 E6/HPV-18 E6- $(\mathrm{n}=154)$. Survival difference was significant $(\mathrm{p}=0.055)$.

difference. Interestingly, a small group of 17 patients with HPV-16 and -18 E6 oncoprotein expression had better survival outcomes than the 154 patients lacking expression of both oncoproteins (Fig. 2); the difference was significant $(\mathrm{p}=0.055)$.

\section{Discussion}

This study used immunohistochemistry to investigate the clinicopathological role of HPV-16/18 E6 oncoprotein expression in patients with pathological stage I NSCLC. The frequency of HPV-16 and -18 E6 oncoprotein expression was 22.6 and $14.3 \%$, respectively, which was less than that of HPV-16 (34.8\%, 50/141) and that of HPV-18 (40.4\%, 58/141) resulting from hybridization in our previous study (8). For prognostic biomarkers with clinical potential, the approach involving HPV tumor status is strongly dependent on the performance of the assay for HPV detection as well as the definition of a positive test (22). Viral oncogene expression is considered the gold standard for establishing the HPV etiology of a tumor in young women with a high-risk of cervical HPV infection and normal cervical cytology (23). Generally, the detection rates of HPV in lung cancers show wide variations, recording 2739 cases described a detection rate of $22.16 \%$ from various studies $(20,24,25)$. Our current study represents the first report of HPV-16/18 E6 oncoprotein expression in patients with pathological stage I NSCLC in Taiwan.

Similar to other studies, our immunohistochemical results demonstrated that HPV-16/18 E6 oncoprotein expression affects the demographics, clinical presentation, and histopathology of lung cancers patients. Patients with HPV-positive head and neck squamous cell carcinoma (HNSCC) tend to be younger, nonsmokers, and nondrinkers; in addition, their tumors tend to arise largely from the lingual and palatine tonsils in the oropharynx (26). Histopatholocally, HPV-positive HNSCC tends to have a poorly differentiated and frequently basaloid histology $(26,27)$. The results from our previous study are similar to the results obtained from the current study and 
indicate that there is a significant association between HPV$16 / 18$ infection and nonsmoking females and patients with adenocarcinoma of the lung (8). The results from both of our studies contrast with those in other studies which show that HPV-16/18 is more prevalent in patients with lung SCC than in patients with lung adenocarncinoma $(13,15)$. The role HPV-16/18 infection plays in the development of lung adenocarcinoma needs to be investigated further (8). Furthermore, multiple logistic regression analyses showed that females, nonsmokers and patients with adenocarcinoma had significantly higher odds of expressing HPV-16 E6 oncoprotein. After adjusting for sex, age, smoking habit, tumor stage and pathology, females (OR, 2.275) and patients adenocarcinoma (OR, 2.320) still had significantly higher ORs. For HPV-18 E6 expression, there were no significant variables after adjusting for sex, age, smoking habit, tumor stage and pathology.

To our knowledge, no studies demonstrating the clinical outcome for HPV-16/18 E6 oncoprotein expression in pathological stage I NSCLC patients have been published. The results from this study revealed that patients with HPV-16 and -18 E6 oncoprotein expression had significantly higher 5-year cumulate survival rates than patients lacking expression of both proteins. Similarly, most previous studies on HNSCC have demonstrated that patients with HPV infection have a better prognosis than HPV-negative patients (27-30).

Ragin and Taioli conducted a meta-analysis on the overall relationship between HPV infection and overall survival and disease-free survival in HNSCC, and found that patients with HPV-positive HNSCC had a lower risk of dying, and a lower risk of recurrence than HPV-negative HNSCC patients (31). The reason for the improved survival is still unclear; however, improved radiation responsiveness, immune surveillance to viral antigens, and the absence of field cancerization in nonsmokers have been postulated (26). In addition, HPVpositive tumors tend to have a wild-type p53 that is functionally inactivated by E6 oncoprotein. Conversely, HPV-negative tumors usually have a specific p53 mutation that can be induced by smoking (26). In our recent study, we investigated the p53 mutation rate in 483 lung cancer patients and found that the frequency of mutation was higher in male smokers and lowest in female nonsmokers (data not shown). Collectively, E6-related degradation of p53 in HPV-positive cancers may be functionally inequivalent to HPV-negative p53 mutations (32,33); and therefore, HPV-positive tumors may have an intact apoptotic response to radiation and chemotherapy (34). Furthermore, a previous study has shown that infection with potentially oncogenic HPV types could co-operate with K-ras gene activation in the progression of the disease, since K-ras activation by point mutations seems to be a late event in lung carcinogenesis (35).

Our recent study regarding p53 mutation and immunostaining results from female lung cancer patients lead us to consider the possibility that HPV infection may be associated with lung tumorigenesis through an HPV E6-dependent p53 degradation pathway, similar to the tumorigenesis of HPVassociated cervical cancer (36). Plausibly, HPV-16/18 infection may partly play a role in lung tumorigenesis in nonsmoking Taiwanese women. In consideration of poorer prognosis of HPV-16- and HPV-18-negative patients with pathological stage I NSCLC, subsequent adjuvant therapy is warranted.

\section{Acknowledgements}

The study was supported in part by research a grant from the National Science Council Taiwan (NSC95-2314-B-039-021), and in part by China Medical University Hospital, Taichung, Taiwan (CMU95-131).

\section{References}

1. Kim JS, Kim JW, Han J, et al: Cohypermethylation of p16 and FHIT promoters as a prognostic factor of recurrence in surgically resected stage I non-small cell lung cancer. Cancer Res 66: 4049-4054, 2006

2. Harpole DH: Prognostic modeling in early stage lung cancer: an evolving process from histopathology to genomics. Thorac Surg Clin 17: 167-173, 2007.

3. Mountain CF: Revisions in the international system for staging lung cancer. Chest 111: 1710-1717, 1997.

4. Slebos RJ, Kibbelaar RE, Dalesio O, et al: K-ras oncogene activation as a prognostic marker in adenocarcinoma of the lung. N Engl J Med 323: 561-565,1990.

5. Herbst RS, Yano S, Kuniyasu H, et al: Differential expression of E-cadherin and type IV collagenase genes predicts outcome in patients with stage I non-small cell lung carcinoma. Clin Cancer Res 6: 790-797, 2000.

6. Pezzella F, Turley H, Kuzu I, et al: bcl-2 protein in non-smallcell lung carcinoma. N Engl J Med 329:690-694,1993.

7. Lee JS, Ro JY, Sahin AA, et al: Expression of blood-group antigen A - a favorable prognostic factor in non-small-cell lung cancer. N Engl J Med 324: 1084-1090,1991.

8. Cheng YW, Chiou HL, Sheu GT, et al: The association of human papillomavirus 16/18 infection with lung cancer among nonsmoking Taiwanese women. Cancer Res 61: 2799-2803, 2001.

9. Gorgoulis VG, Zacharatos P, Kotsinas A, et al: Human papillomavirus (HPV) is possibly involved in laryngeal but not in lung carcinogenesis. Hum Pathol 30: 274-283, 1990.

10. Clavel CE, Nawrocki B, Bosseaux B, et al: Detection of human papillomavirus DNA in bronchopulmonary carcinomas by hybrid capture II: a study of 185 tumors. Cancer 88: 1347-1352, 2000.

11. Coissard CJ, Besson G, Polette MC, et al: Prevalence of human papillomaviruses in lung carcinomas: a study of 218 cases. Mod Pathol 18: 1606-1609, 2005.

12. Tsuhako K, Nakazato I, Hirayasu T, et al: Human papillomavirus DNA in adenosquamous carcinoma of the lung. J Clin Pathol 51: 741-749, 1998.

13. Nadji SA, Mokhtari-Azad T, Mahmoodi M, et al: Relationship between lung cancer and human papillomavirus in north of Iran, Mazandaran province. Cancer Lett 248: 41-46, 2007.

14. Soini Y, Nuorva K, Kamel D, et al: Presence of human papillomavirus DNA and abnormal p53 protein accumulation in lung carcinoma. Thorax 51: 887-893,1996.

15. Hirayasu T, Iwamasa T, Kamada Y, et al: Human papillomavirus DNA in squamous cell carcinoma of the lung. J Clin Pathol 49: 810-817, 1996.

16. Werness BA, Levine AJ and Howley PM: Association of human papillomavirus types 16 and 18 E6 proteins with $\mathrm{p} 53$. Science 248: 76-79, 1990

17. Scheffner M, Werness BA, Huibregtse JM, et al: The E6 oncoprotein encoded by human papillomavirus types 16 and 18 promotes the degradation of p53. Cell 63: 1129-1136, 2000.

18. Kinshoshita I, Dosaka-Akita H, Shindoh M, et al: Human papillomavirus type 18 DNA and E6-E7 mRNA are detected in squamous cell carcinoma and adenocarcinoma of the lung. Br J Cancer 71: 344-349, 1995.

19. Ciotti M, Giuliani L, Ambrogi V, et al: Detection and expression of human papillomavirus oncogenes in non-small cell lung cancer. Oncol Rep 16: 183-189, 2006.

20. Brouchet L, Valmary S, Dahan M, et al: Detection of oncogenic virus genomes and gene products in lung carcinoma. Br J Cancer 92: 743-746, 2005.

21. WHO: The World Health Organization histological typing of lung tumors. Am J Clin Pathol 77: 123-136, 1982.

22. McShane LM, Altman DG and Sauerbrei W: Identification of clinically useful cancer prognostic factors: what are we missing. J Natl Cancer Inst 97: 1023-1025, 2005. 
23. Molden T, Kraus I, Kalsen F, et al: Human papillomarirus E6/E7 mRNA expression in women younger that 30 years of age. Gynecol Oncol 100: 95-100, 2006.

24. Syrjanen KJ: HPV infections and lung cancer. J Clin Pathol 55: 885-891, 2002.

25. Chiou HL, Wu MF, Liaw YC, et al: The presence of human papillomavirus type 16/18 DNA in blood circulation may act as a risk marker of lung cancer in Taiwan. Cancer 97: 1558-1563, 2003.

26. Fakhry $\mathrm{C}$ and Gillison ML: Clinical implications of human papillomavirus in head and neck cancers. J Clin Oncol 24: 2606-2611, 2006.

27. Gillison ML, Koch WM, Capone RB, et al: Evidence for a causal association between human papillomavirus and a subset of head and neck cancers. J Natl Cancer Inst 92: 709-720, 2000.

28. Ritchie JM, Smith EM, Summersgill KF, et al: Human papillomavirus infection as a prognostic factor in carcinomas of the oral cavity and oropharynx. Int J Cancer 104: 336-344, 2003.

29. Lindel K, Beer KT, Laissue J, et al: Human papillomavirus positive squamous cell carcinoma of the oropharynx: a radiosensitive subgroup of head and neck carcinoma. Cancer 92: 805-813, 2001.

30. Weinberger PM, Yu Z, Haffty BG, et al: Molecular classification identifies a subset of human papillomavirus-associated oropharyngeal cancers with favorable prognosis. J Clin Oncol 24: 736-747, 2006.
31. Ragin CRC and Taioli E: Survival of squamous cell carcinoma of the head and neck in relation to human papillojavirus infection: review and meta-analysis. Int J Cancer 121: 1813-1820, 2007.

32. Butz K, Whitaker N, Denk C, et al: Induction of the p53-target gene GADD45 in HPV-positive cancer cells. Oncogene 18: 2381-2386, 1999.

33. Huang H, Li CY and Little JB: Abrogation of P53 function by transfection of HPV16 E6 gene does not enhance resistance of human tumor cells to ionizing radiation. Int J Radiat Biol 170: 151-160, 1996.

34. Deweese TL, Walsh JC, Dillehay LE, et al: Human papillomavirus E6 and E7 oncoproteins alter cell cycle progression but not radiosensitivity of carcinoma cells treated with low-doserate radiation. Int J Radiat Oncol Biol Phys 37: 145-154, 1997.

35. Noutsou A, Koffa M, Ergazaki M, et al: Detection of human papilloma virus (HPV) and K-ras mutations in human lung carcinomas. Int J Oncol 8: 1089-1093, 1996.

36. Cheng YW, Wu MF, Wang J, et al: Human papillomavirus $16 / 18$ E6 oncoprotein is expressed in lung cancer and related with p53 inactivation. Cancer Res 67: 1-8, 2007. 